Supplement of Hydrol. Earth Syst. Sci., 25, 1009-1032, 2021

https://doi.org/10.5194/hess-25-1009-2021-supplement

(c) Author(s) 2021. This work is distributed under

the Creative Commons Attribution 4.0 License.

(c) (1)

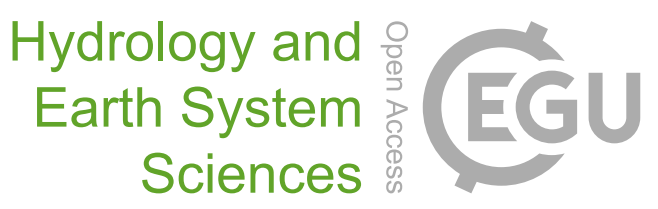

Supplement of

\title{
Lake thermal structure drives interannual variability in summer anoxia dynamics in a eutrophic lake over 37 years
}

Robert Ladwig et al.

Correspondence to: Robert Ladwig (rladwig2@wisc.edu)

The copyright of individual parts of the supplement might differ from the CC BY 4.0 License. 


\section{Supplementary material}

\section{PIHM-Lake description}

PIHM-Lake is built upon a physically-based spatially distributed hydrologic model—PIHM (Penn State Integrated

5 Hydrologic Model) (Qu and Duffy, 2007) — with the capability of simulating surface, subsurface, and channel water exchange between a catchment and a lake, as well as the water level change of the lake. As illustrated in Supplement Figure S11, PIHMLake model uses a finite volume numerical scheme and unstructured triangular mesh to represent the domain. It tracks the changes of surface and subsurface water storage on a 3D catchment and 1D lake as a function of precipitation, evapotranspiration, recharge, surface and groundwater flow, channelized flow, and snow melt. The spatial variation of overland

10 flow and groundwater flow between the catchment and the lake is characterized by the water flows through the edges of each triangular mesh. Specifically, based on the conservation of mass of water, the generic form of the governing equations for PIHM-Lake is

$$
\left\{\begin{aligned}
\frac{d S_{\text {canopy }}}{d t} & =v F r a c *(1-s F r a c) * P-E_{c} \\
\frac{d S_{\text {snow }}}{d t} & =s F r a c * P-S M \\
\frac{\partial S_{\text {surf }}}{\partial t} & =T F-\nabla q_{\text {sw }}-I-E_{s} \\
\frac{d S_{\text {unsat }}}{d t} & =I-R-E_{g}-E_{g t} \\
\frac{\partial S_{\text {sat }}}{\partial t} & =-\nabla q_{g w}+R-E_{\text {sat }}-E_{\text {tsat }}
\end{aligned}\right.
$$

15 where $\frac{d S_{\text {canopy }}}{d t}=$ the time rate of change of the canopy water storage, $S_{\text {canopy }}(\mathrm{m})$, due to canopy evaporation $E_{c}(\mathrm{~m} / \mathrm{day})$ and canopy interception $v F r a c *(1-s F r a c) * P(\mathrm{~m} /$ day $)$. vFrac and $s F r a c$ are the vegetation fraction and snow fraction, respectively. $P=$ precipitation $\left(\mathrm{m} /\right.$ day). $\frac{d S_{\text {snow }}}{d t}=$ the time rate of change of snow storage $S_{\text {snow }}(\mathrm{m})$ due to $s F r a c * P$ :snow formation from precipitation when temperature is below $0{ }^{\circ} \mathrm{C}(\mathrm{m} /$ day) and $S M$, snow melt $(\mathrm{m} /$ day), which is a function of degree-day factor of ice and snow melt. $\frac{\partial S_{\text {surf }}}{\partial t}=$ the time rate of change of surface water storage, $S_{\text {surf }}(\mathrm{m})$, due to $T F=$ 
20 throughfall (m/day), $\nabla q_{s w}=$ net overland flow (m/day), $I$ : infiltration (m/day), and $E_{s}$ : surface water evaporation (m/day). $\nabla q_{s w}$ is modeled by the diffusion wave approximation of St. Venant's equation assuming shallow surface water depth and negligible influence of inertia force on overland flow, which is equivalent to Manning's equation. The estimation of infiltration rate is a function of the gradient of the surface and subsurface hydraulic head. $\frac{d s_{\text {unsat }}}{d t}$ represents the time rate of change of unsaturated water storage $(\mathrm{m})$ due to $I$ : infiltration (m/day), $R$ : recharge (m/day), $E_{g}:$ soil evaporation (m/day), and $E_{g t}$ :

25 transpiration (m/day). The recharge is calculated using Richard's equation assuming a vertical exchange of water across a moving water table interface. $\frac{\partial S_{s a t}}{\partial t}=$ the time rate of change of $S_{s a t}$ : the saturated water storage $(\mathrm{m}) . \nabla q_{g w}=$ net groundwater lateral movement between adjacent cells $(\mathrm{m} / \mathrm{day})$ is represented by the Darcy-type flow proportional to groundwater gradient. $E_{c}, E_{s}, E_{g}$ and $E_{s a t}$ are the evaporation (m/day) from the vegetation canopy, surface water, unsaturated and saturated soil zone, respectively. The potential evaporation rate is estimated by the Penman equation. The transpiration (m/day) is

30 described by $E_{g t}$ or $E_{t s a t}$, depending upon the vegetation coverage, the rooting depth and the groundwater table. If the groundwater table is higher than rooting depth, plants uptake water from the saturated zone, and $E_{t s a t}$ applies. Otherwise, water uptake occurs at the unsaturated soil zone, and $E_{g t}$ applies.

For the hydrodynamics of the 1-D lake, we consider a two-layer system: a surface water layer and an aquifer layer. Surface water flow between the catchment boundary cells directly affects the water storage of surface water layer. Meanwhile,

35 subsurface water flows through the aquifer layer and indirectly contributes to surface water through negative recharge. Likewise, based on the conservation of mass of water, the governing equation for the 1D lake component is

$$
\left\{\begin{array}{c}
\frac{\partial S_{\text {lake_surf }}}{\partial t}=P-E_{S}-R+q_{s w} \\
\frac{\partial S_{\text {lake_gw }}}{\partial t}=R+q_{g w}
\end{array}\right.
$$

where $\frac{\partial S_{\text {lake_surf }}}{\partial t}=$ the time rate of change of lake surface water. $\frac{\partial S_{\text {lake } g w}}{\partial t}=$ the time rate of change of water storage in lake bottom aquifer. $P=$ precipitation $(\mathrm{m} /$ day $) ; E_{s}=$ surface water evaporation $(\mathrm{m} /$ day $) ; R=$ recharge $(\mathrm{m} /$ day $)$. A positive value of

$40 \mathrm{R}$ indicates downward lake surface water, while a negative value indicates an upward groundwater recharge to surface water; $q_{s w}$ and $q_{g w}$ are surface and groundwater flow through the edges of the lake boundary, respectively. 
Details of the model processes and code is referred to the model repository: https://github.com/hydro-geomorph-zhang/PIHMLake.

Figures
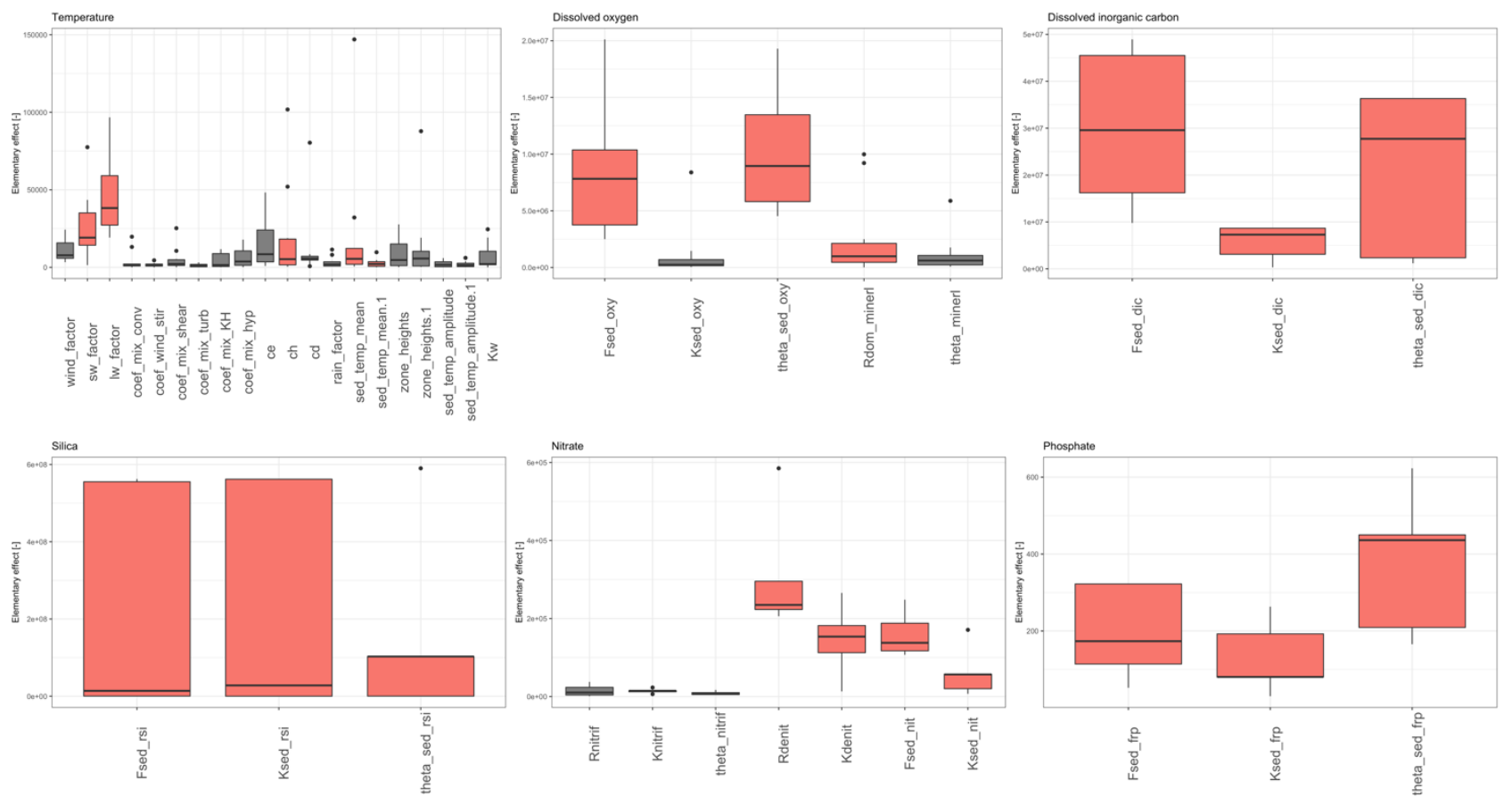

Figure S1 Mean and standard deviations of absolute elementary effects quantified by the Morris Method for water temperature, dissolved oxygen, dissolved inorganic carbon, silica, nitrate and phosphate. Colored bars are sensitive parameters that were used in the calibration. 
(b) Water temperature [deg C], $10 \mathrm{~m}$ : RMSE 2.08deg C NSE 0.89 KGE 0.93

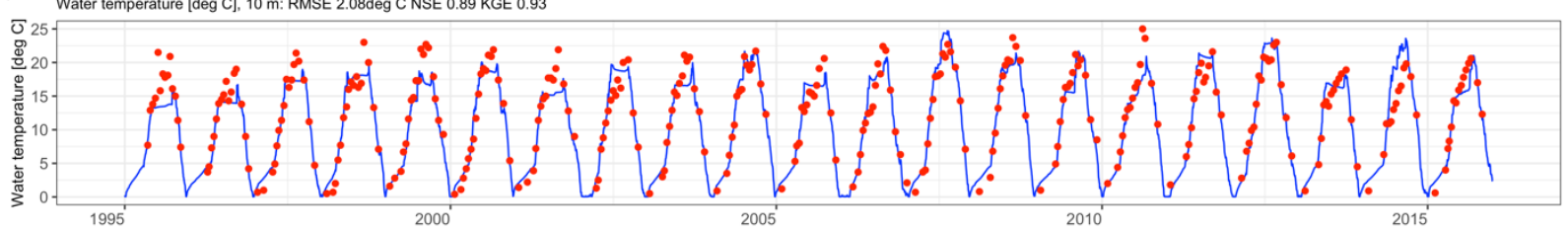

(c)

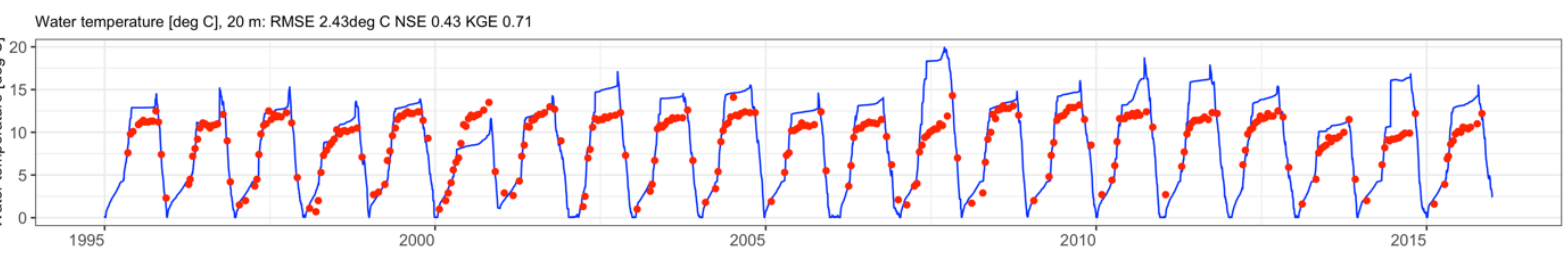

50 Figure S2 Time-series comparison between observed (red dots) and water temperatures (blue lines). The fit criteria root-mean square error (RMSE), Nash-Sutcliffe coefficient of efficiency (NSE) and Kling-Gupta coefficient of efficien
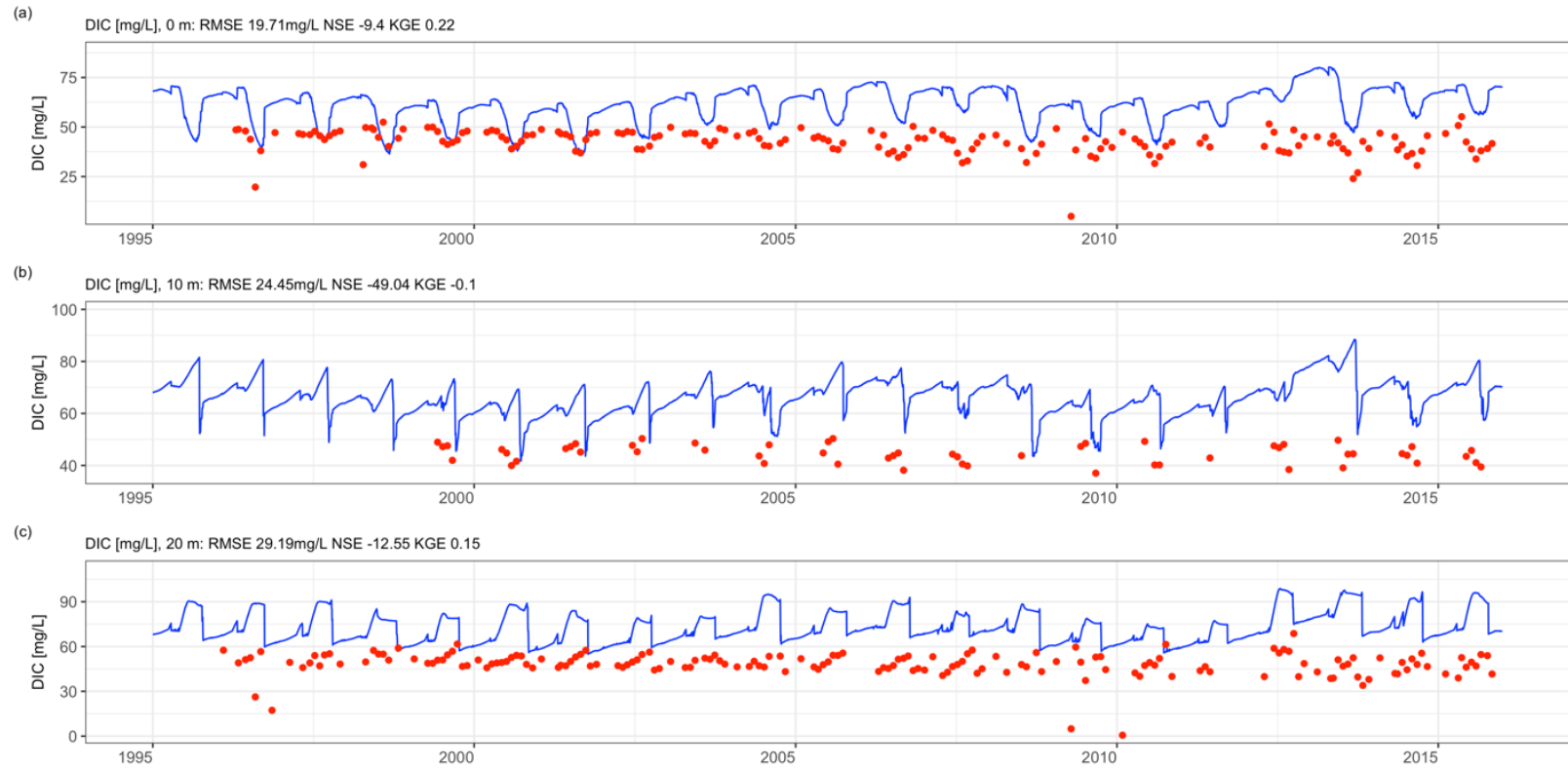

Figure S3 Time-series comparison between observed (red dots) and modeled dissolved inorganic carbon concentrations (blue lines). The fit criteria root-mean square error (RMSE), Nash-Sutcliffe coefficient of efficiency (NSE) and Kling-Gupta coefficient of efficiency (KGE). 

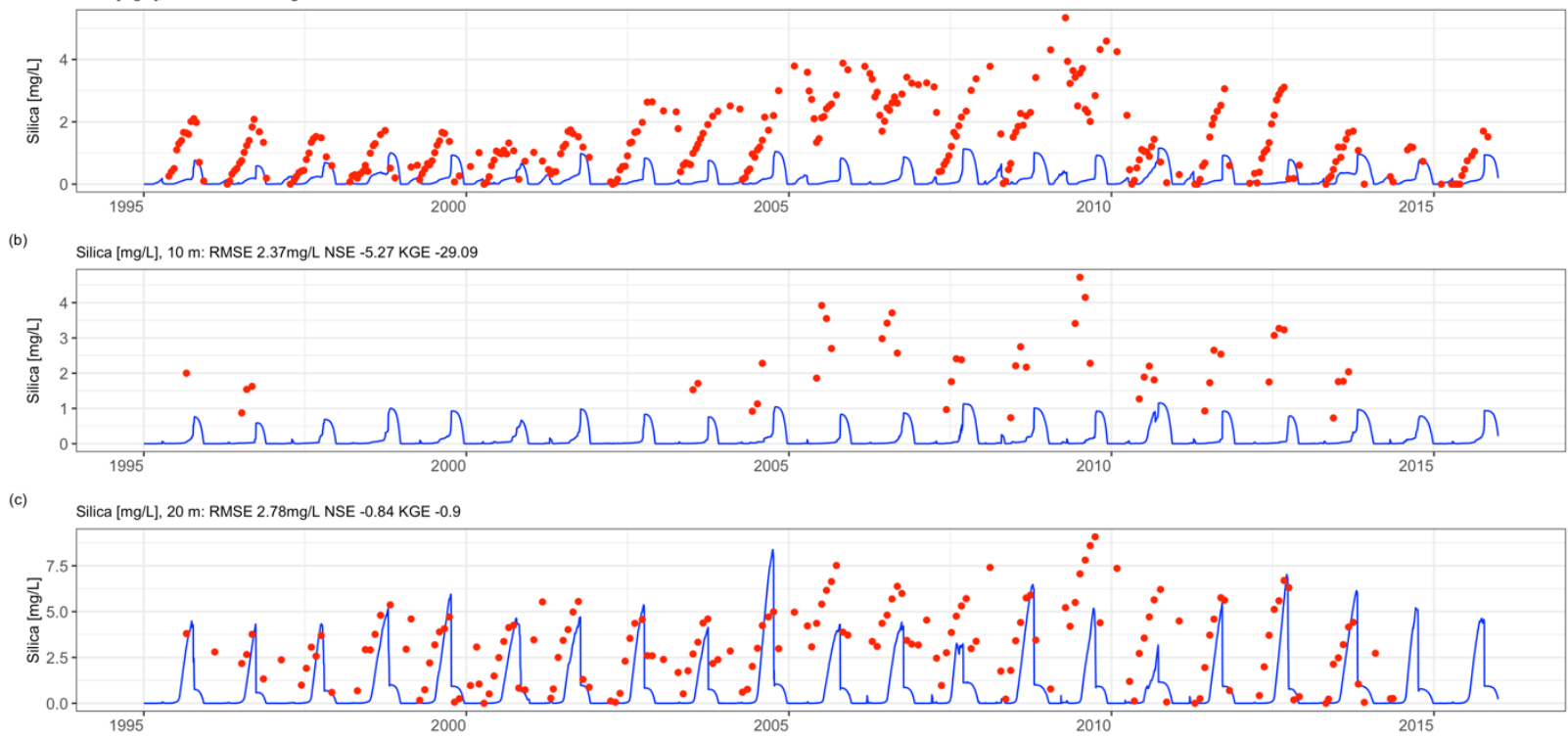

Figure S4 Time-series comparison between observed (red dots) and modeled silica concentrations (blue lines). The fit criteria rootmean square error (RMSE), Nash-Sutcliffe coefficient of efficiency (NSE) and Kling-Gupta coefficient of efficiency (KGE).
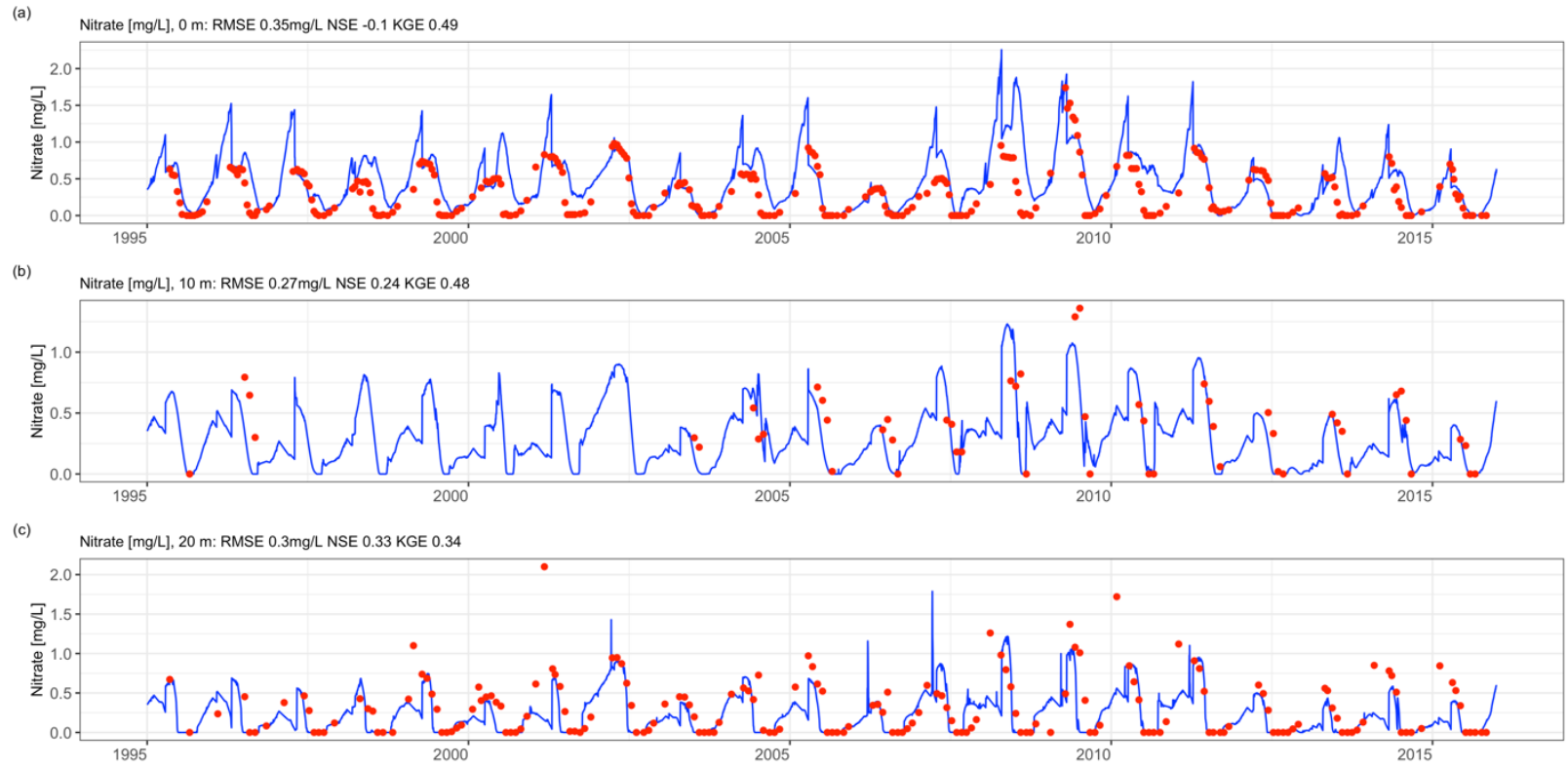

60 Figure S5 Time-series comparison between observed (red dots) and modeled nitrate concentrations (blue lines). The fit criteria rootmean square error (RMSE), Nash-Sutcliffe coefficient of efficiency (NSE) and Kling-Gupta coefficient of efficiency (KGE). 
(a)

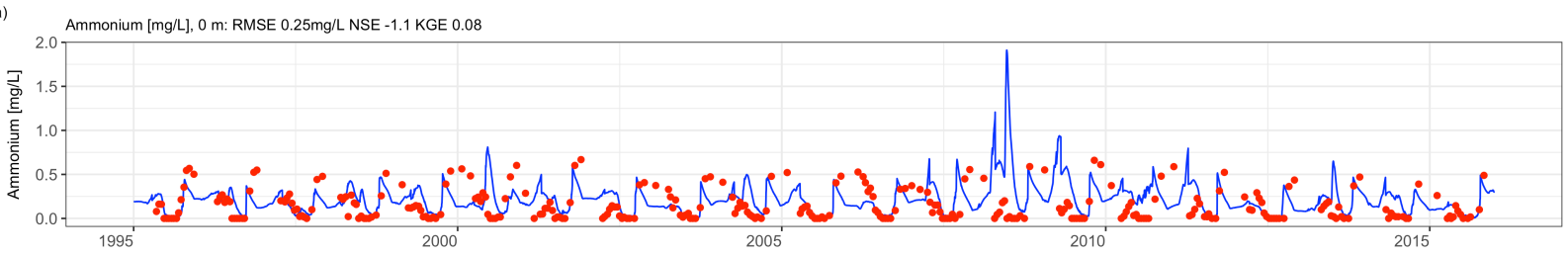

(b)

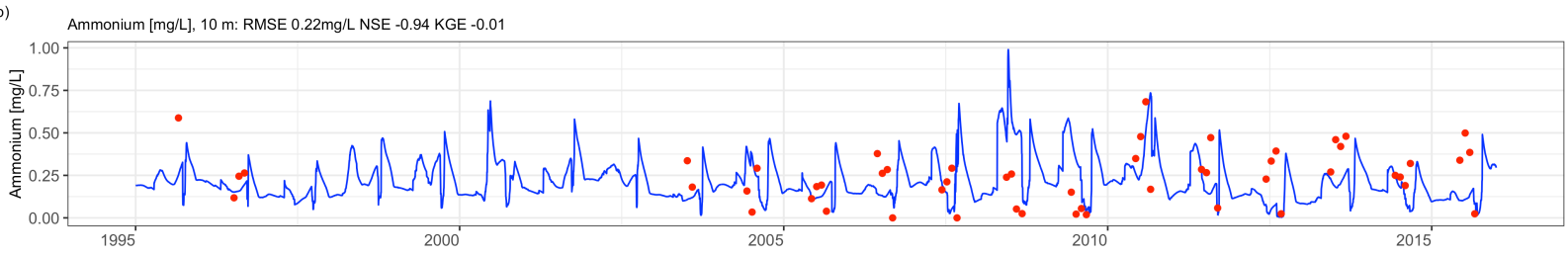

(c)

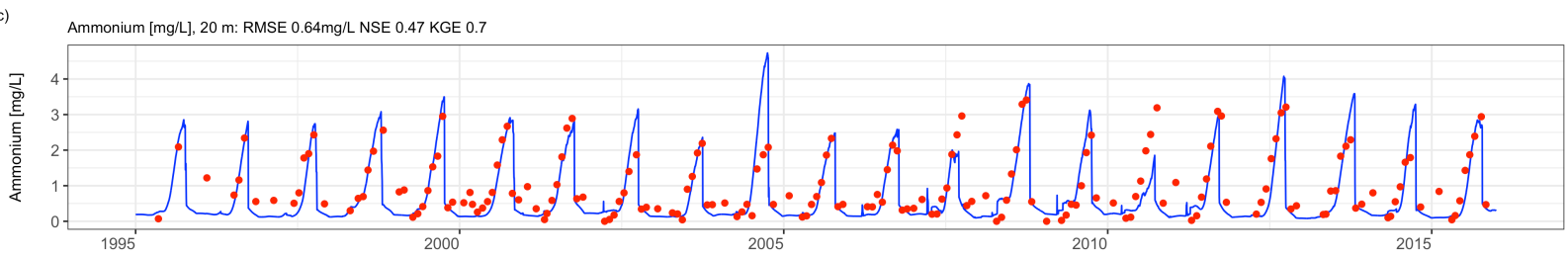

Figure S6 Time-series comparison between observed (red dots) and modeled ammonium concentrations (blue lines). The fit criteria root-mean square error (RMSE), Nash-Sutcliffe coefficient of efficiency (NSE) and Kling-Gupta coefficient of efficiency (KGE).

(a)

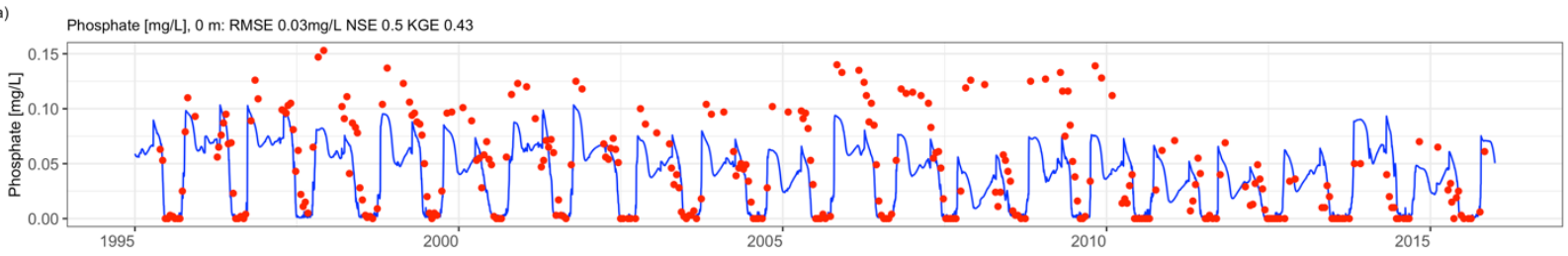

(b)

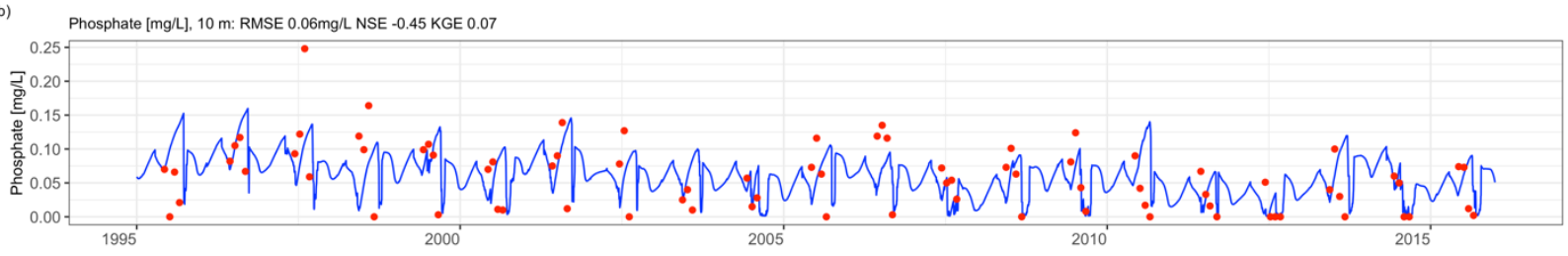

(c)

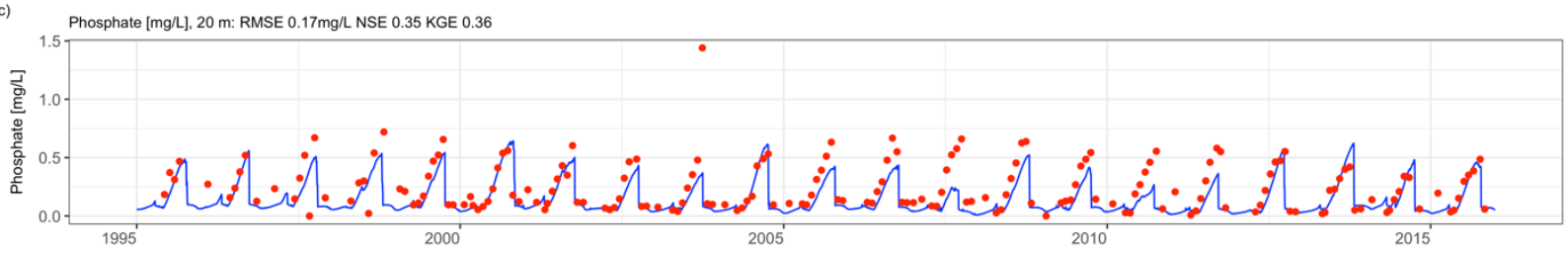

Figure S7 Time-series comparison between observed (red dots) and modeled phosphate concentrations (blue lines). The fit criteria root-mean square error (RMSE), Nash-Sutcliffe coefficient of efficiency (NSE) and Kling-Gupta coefficient of efficiency (KGE). 
(a)
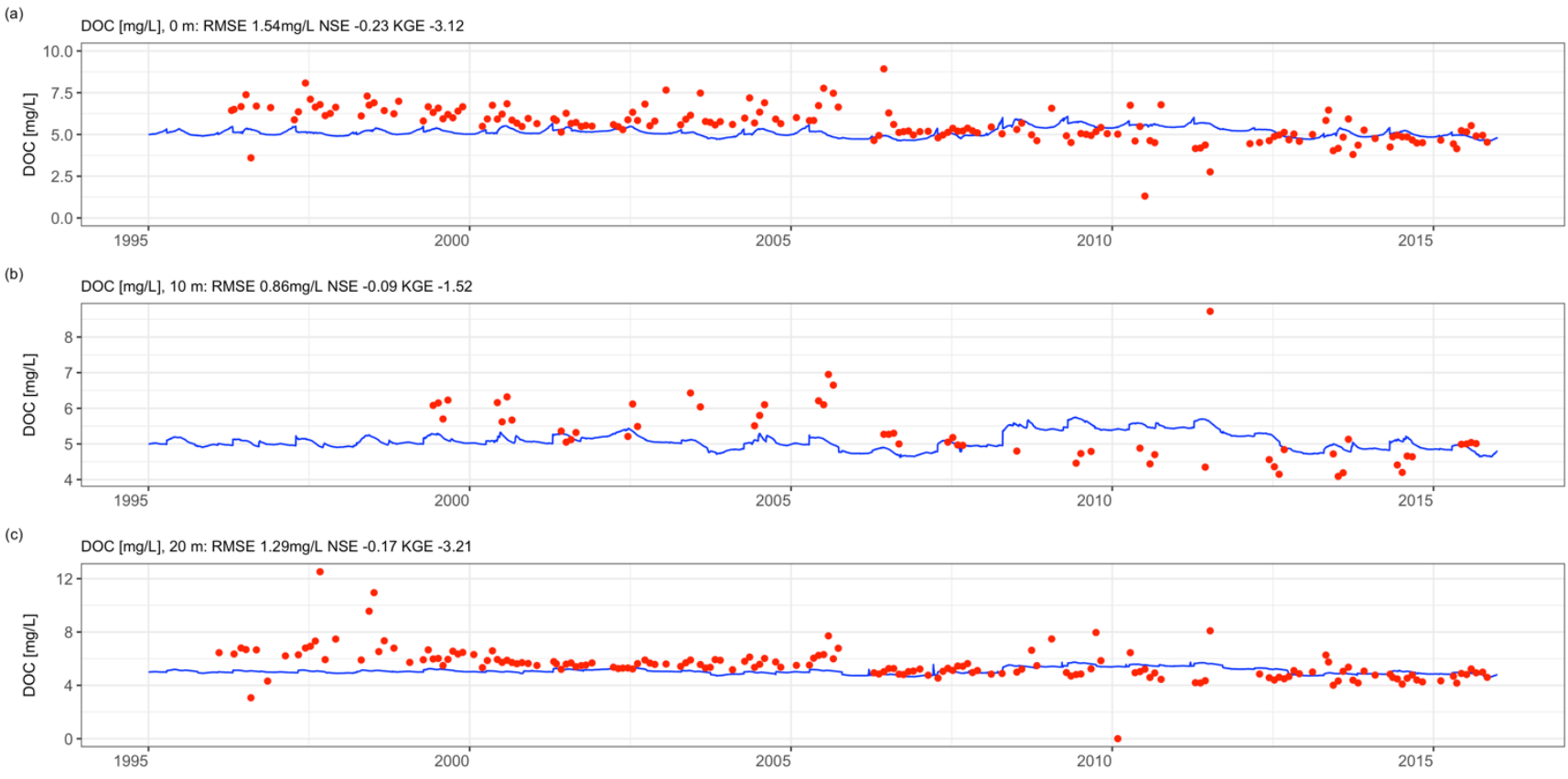

Figure S8 Time-series comparison between observed (red dots) and modeled dissolved organic carbon concentrations (blue lines).

70 The fit criteria root-mean square error (RMSE), Nash-Sutcliffe coefficient of efficiency (NSE) and Kling-Gupta coefficient of efficiency (KGE). 
(a)

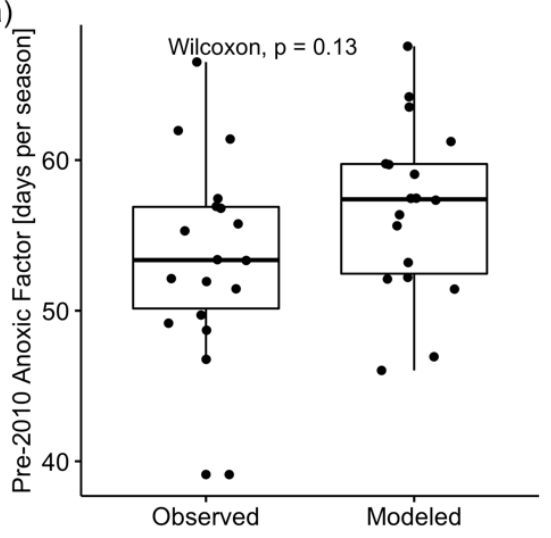

(c)

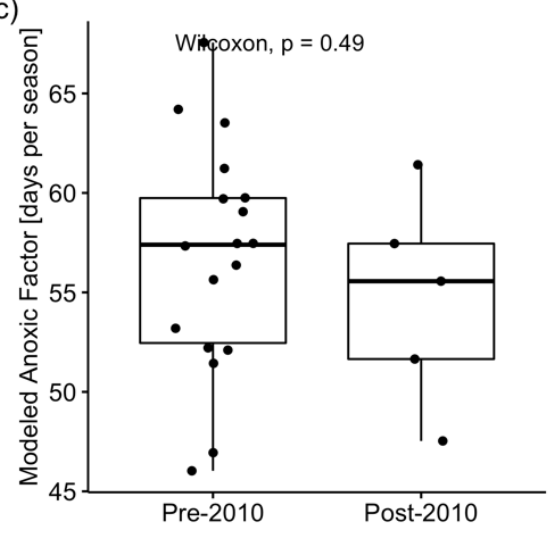

(b)

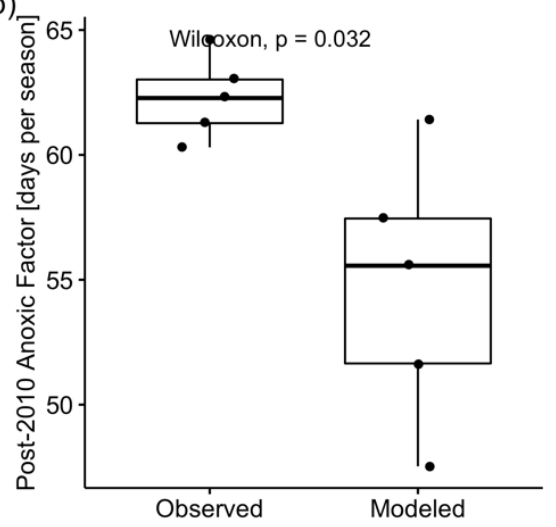

(d)

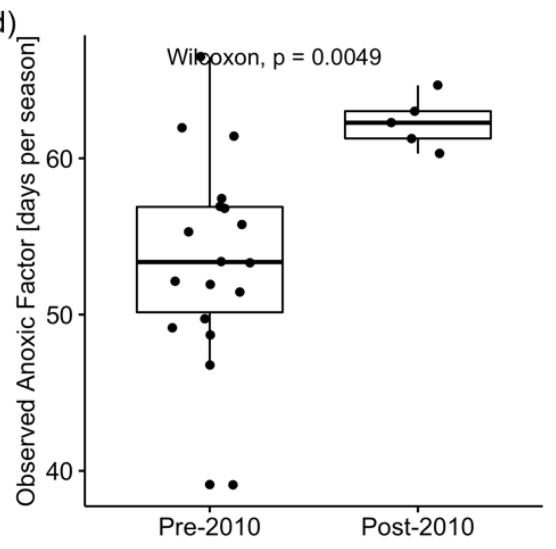

Figure S9 Box-whisker plots of (a) observed to modeled Anoxic Factors for the pre-2010 period 1992-2009, (b) observed to modeled 75 Anoxic Factors for the post-2010 period 2010-2015, (c) pre- to post-2010 modeled Anoxic Factors, and (d) pre- to post-2010 observed Anoxic Factors. 
(a)

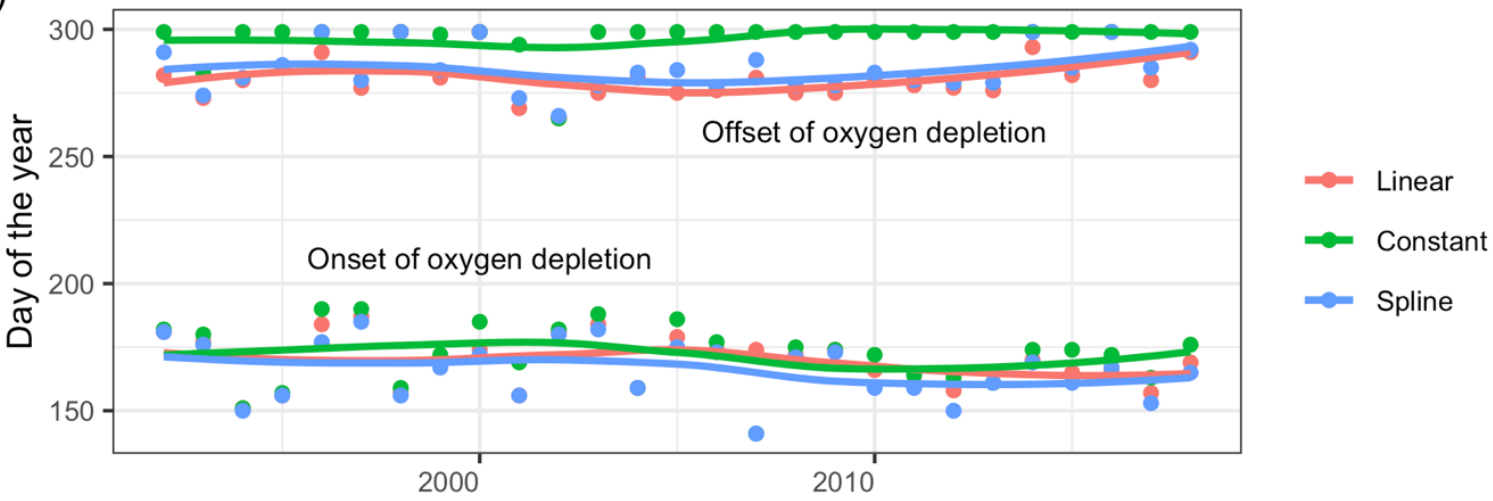

(b)

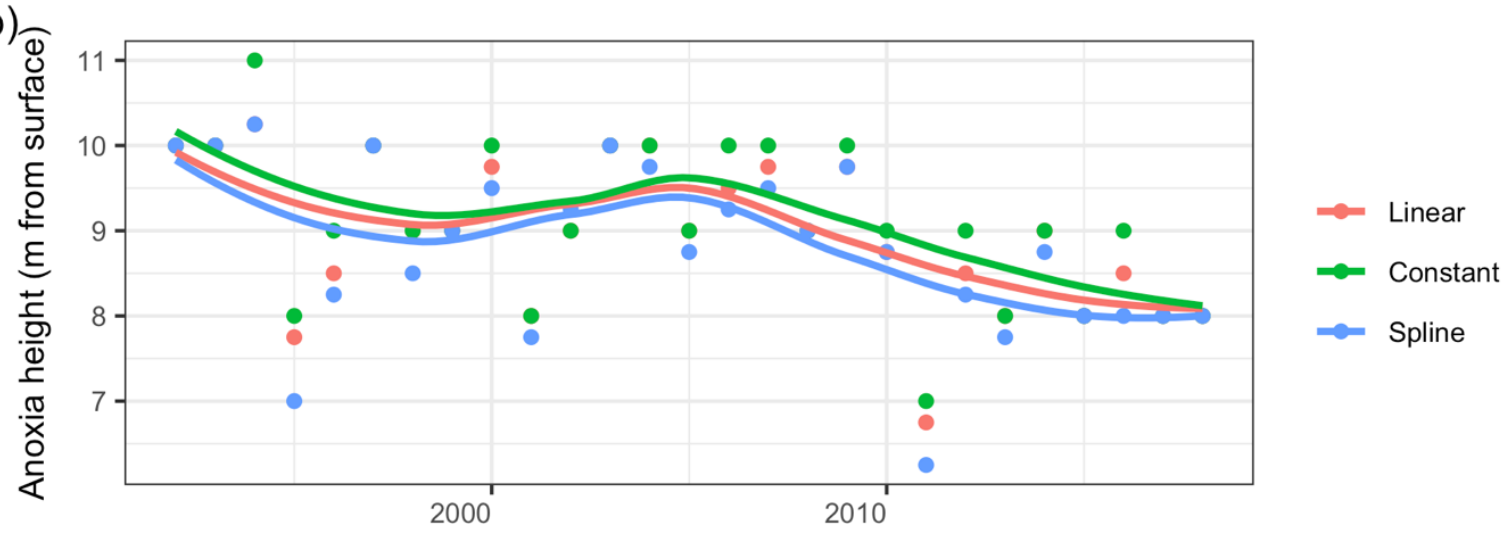

Figure S10 Observed anoxia onset, offset (a) and height (b) dynamics. The colored lines refer to the interpolation method. 


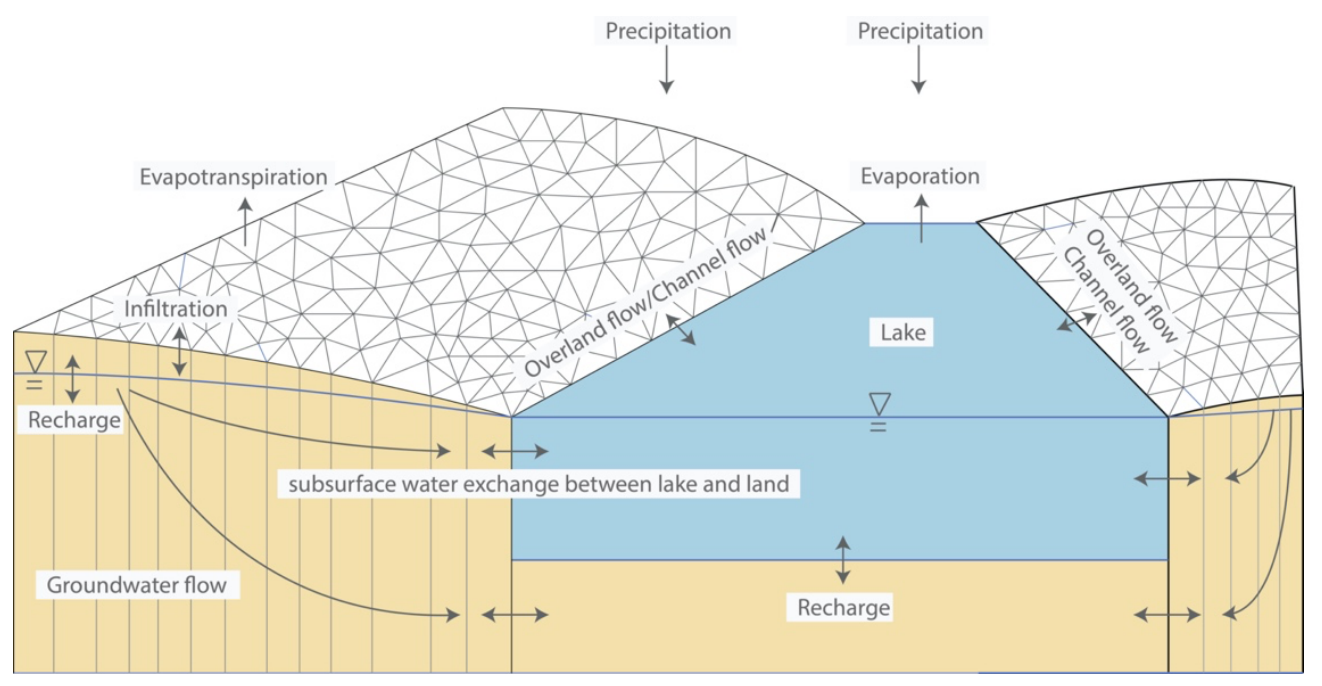

80 Figure S11 Conceptual framework of PIHM-Lake.

\section{Tables}

Table S1 Model parameters for functional phytoplankton groups

\begin{tabular}{|c|c|c|c|}
\hline Parameter & Description & Cyanobacteria & Diatoms \\
\hline P_initial & $\begin{array}{l}\text { Initial concentration of phytoplankton (mmol } \\
\mathrm{C} / \mathrm{m3} \text { ) }\end{array}$ & 10 & 8.4 \\
\hline PO & $\begin{array}{l}\text { Minimum concentration of phytoplankton } \\
\text { (mmol C/m3) }\end{array}$ & 0.03 & 0.03 \\
\hline W_p & sedimentation rate $(\mathrm{m} / \mathrm{d})$ & 0 & -0.05 \\
\hline Xcc & carbon to chlorophyll ratio (mg C/mg chla) & 50 & 50 \\
\hline R_growth & Phyto max growth rate @20c (/day) & 0.8 & 2.8 \\
\hline fT_Method & Temperature limitation function of growth & CAEDYM style & CAEDYM style \\
\hline Theta_growth & $\begin{array}{l}\text { Arrhenius temperature scaling for growth } \\
\text { function (-) }\end{array}$ & 1.06 & 1.06 \\
\hline T_std & Standard temperature ( $\operatorname{deg} \mathrm{C})$ & 20 & 15 \\
\hline T_opt & Optimum temperature (deg C) & 28 & 20 \\
\hline T_max & Maximum temperature (deg C & 35 & 32 \\
\hline lightModel & Type of light response function & no photoinhibition & no photoinhibition \\
\hline I_K & $\begin{array}{l}\text { Half saturation constant for light limitation of } \\
\text { growth (microE } / \mathrm{m}^{\wedge} 2 / \mathrm{s} \text { ) }\end{array}$ & 25 & 10 \\
\hline
\end{tabular}




\begin{tabular}{|c|c|c|c|}
\hline KePHY & $\begin{array}{l}\text { Specific attenuation coefficient }((\mathrm{mmol} C \\
\left.\left.\mathrm{m}^{\wedge} 3^{\wedge}-1\right)^{\wedge} 1 \mathrm{~m}^{\wedge}-1\right)\end{array}$ & 0.005 & 0.001 \\
\hline F_pr & $\begin{array}{l}\text { Fraction of primary production lost to } \\
\text { exudation (-) }\end{array}$ & 0.005 & 0.002 \\
\hline R_resp & $\begin{array}{l}\text { Phytoplankton respiration/metabolic loss } \\
\text { rate @ } 20(\operatorname{deg} C)\end{array}$ & 0.08 & 0.12 \\
\hline Theta_resp & $\begin{array}{l}\text { Arrhenius temperature scaling factor for } \\
\text { respiration (-) }\end{array}$ & 1.05 & 1.07 \\
\hline K_fres & $\begin{array}{l}\text { Fraction of metabolic loss that is true } \\
\text { respiration (-) }\end{array}$ & 0.6 & 0.6 \\
\hline K_fdom & Fraction of metabolic loss that is DOM (-) & 0.05 & 0.05 \\
\hline simDINUptake & Simulate DIN uptake & True & True \\
\hline simINDynamics & Simulate internal $\mathrm{N}$ & Fixed C:N & Dynamic C:N \\
\hline N_0 & $\begin{array}{l}\text { Nitrogen concentration below which uptake } \\
\text { is } 0\left(\mathrm{mmol} \mathrm{N} / \mathrm{m}^{\wedge} 3\right)\end{array}$ & 0 & 0 \\
\hline K_N & $\begin{array}{l}\text { Half-saturation concentration of nitrogen } \\
\left(\mathrm{mmol} \mathrm{N} / \mathrm{m}^{\wedge} 3\right)\end{array}$ & 1 & 3.5 \\
\hline X_ncon & $\begin{array}{l}\text { Constant internal nitrogen concentration } \\
\text { ( } \mathrm{mmol} \mathrm{N} / \mathrm{mmol} \mathrm{C})\end{array}$ & 0.035 & 0.035 \\
\hline X_nmin & $\begin{array}{l}\text { minimum internal nitrogen concentration } \\
\text { ( } \mathrm{mmol} \mathrm{N} / \mathrm{mmol} \mathrm{C})\end{array}$ & 0.06 & 0.077 \\
\hline X_nmax & $\begin{array}{l}\text { maximum internal nitrogen concentration } \\
\text { ( } \mathrm{mmol} \mathrm{N} / \mathrm{mmol} \mathrm{C})\end{array}$ & 0.206 & 0.129 \\
\hline R_nuptake & $\begin{array}{l}\text { maximum nitrogen uptake rate (mmol } \\
\left.\mathrm{N} / \mathrm{m}^{\wedge} 3 / \mathrm{d}\right)\end{array}$ & 0.068 & 0.13 \\
\hline R_nfix & nitrogen fixation rate $(\mathrm{mmol} \mathrm{N} / \mathrm{mmol} \mathrm{C} /$ day) & 0.13 & 0 \\
\hline simDIPUptake & Simulate DIP uptake & True & True \\
\hline simIPDynamics & Simulate internal phosphorus dynamics & Dynamic C:P & Dynamic C:P \\
\hline P_0 & $\begin{array}{l}\text { Phosphorus concentration below which } \\
\text { uptake is } 0\left(\mathrm{mmol} \mathrm{P} / \mathrm{m}^{\wedge} 3\right)\end{array}$ & 0 & 0 \\
\hline K_P & $\begin{array}{l}\text { Half-saturation concentration of phosphorus } \\
\left(\mathrm{mmol} \mathrm{P} / \mathrm{m}^{\wedge} 3\right)\end{array}$ & 0.5 & 0.7 \\
\hline X_pmin & $\begin{array}{l}\text { Minimum internal phosphorus concentration } \\
\text { (mmol P/mmol C) }\end{array}$ & 0.0019 & 0.0081 \\
\hline
\end{tabular}




\begin{tabular}{|c|c|c|c|}
\hline X_pmax & $\begin{array}{l}\text { Maximum internal phosphorus concentration } \\
\text { (mmol P/mmol C) }\end{array}$ & 0.0089 & 0.033 \\
\hline R_puptake & $\begin{array}{l}\text { Maximum phosphorus uptake rate }(\mathrm{mmol} \\
\left.\mathrm{P} / \mathrm{m}^{\wedge} 3 / \mathrm{d}\right)\end{array}$ & 0.0039 & 0.007 \\
\hline simSIUptake & Simulate Si uptake & False & True \\
\hline Si_0 & $\begin{array}{l}\text { Silica concentration below which uptake is } 0 \\
\left(\mathrm{mmol} \mathrm{Si} / \mathrm{m}^{\wedge} 3\right.\end{array}$ & - & 0 \\
\hline K_Si & $\begin{array}{l}\text { Half-saturation concentration of silica ( } \mathrm{mmol} \\
\mathrm{Si} / \mathrm{m} 3 \text { ) }\end{array}$ & - & 2.5 \\
\hline X_sicon & $\begin{array}{l}\text { Constant internal silica concentration (mmol } \\
\mathrm{Si} / \mathrm{mmol} \mathrm{C} \text { ) }\end{array}$ & - & 0.04 \\
\hline
\end{tabular}

\section{Table S2 Calibrated model parameters}

\begin{tabular}{|c|c|c|c|c|}
\hline Parameter & Description & Unit & $\begin{array}{l}\text { Default value } \\
\text { (Hipsey et al., } \\
\text { 2019a, 2019b) }\end{array}$ & Model value \\
\hline$f_{S W}$ & Solar radiation scaling factor & - & 1.0 & 0.84 \\
\hline$f_{l w}$ & Long-wave radiation scaling factor & - & 1.0 & 0.99 \\
\hline$C_{H}$ & Bulk aerodynamic coefficient for sensible heat transfer & - & 0.0013 & 0.0014 \\
\hline$T_{Z=1, \text { mean }}$ & Annual mean temperature of the upper sediment zone & ${ }^{\circ} \mathrm{C}$ & - & 5.07 \\
\hline$T_{z=2, \text { mean }}$ & Annual mean temperature of the lower sediment zone & ${ }^{\circ} \mathrm{C}$ & - & 13.47 \\
\hline \multirow[t]{3}{*}{$F_{\max }^{o x y}$} & Max. sediment flux for dissolved oxygen & & -100.0 & -100.0 \\
\hline & & mmol & & \\
\hline & & $\overline{m^{2} d^{2}}$ & & \\
\hline$K_{\text {sed }}^{\text {oxy }}$ & $\begin{array}{l}\text { Half-saturation concentration controlling oxygen } \\
\text { sediment flux }\end{array}$ & $\frac{m m o l}{m^{3}}$ & 50.0 & 15.0 \\
\hline$\theta_{\text {sed }}^{\text {oxy }}$ & Temperature multiplier for oxygen sediment flux & - & 1.0 & 1.08 \\
\hline
\end{tabular}




\begin{tabular}{|c|c|c|c|c|}
\hline$R_{\text {mineral }}^{\text {dom }}$ & $\begin{array}{l}\text { Maximum rate of aerobic mineralisation of labile } \\
\text { dissolved organic matter at } 20^{\circ} \mathrm{C}\end{array}$ & $d^{-1}$ & 0.5 & 0.5 \\
\hline$F_{\max }^{\text {dic }}$ & Max. sediment flux for dissolved inorganic carbon (DIC) & $\frac{m m o l}{m^{2} d^{2}}$ & 4.0 & 250.0 \\
\hline$K_{\text {sed }}^{\text {dic }}$ & $\begin{array}{l}\text { Half-saturation concentration controlling DIC sediment } \\
\text { flux }\end{array}$ & $\frac{m m o l}{m^{3}}$ & 30.0 & 7.0 \\
\hline$\theta_{\text {sed }}^{\text {dic }}$ & Arrhenius temperature multiplier for DIC sediment flux & - & 1.0 & 1.08 \\
\hline$F_{\max }^{r s i}$ & Max. sediment flux for reactive silica & $\frac{m m o l}{m^{2} d^{2}}$ & - & 16.42 \\
\hline$K_{\text {sed }}^{r s i}$ & $\begin{array}{l}\text { Half-saturation concentration controlling silica } \\
\text { sediment flux }\end{array}$ & $\frac{m m o l}{m^{3}}$ & 50.0 & 1.90 \\
\hline$\theta_{\text {sed }}^{r s i}$ & $\begin{array}{l}\text { Arrhenius temperature multiplier for silica sediment } \\
\text { flux }\end{array}$ & - & 1.0 & 1.08 \\
\hline$R_{\text {nitrif }}$ & Maximum rate of nitrification at $20^{\circ} \mathrm{C}$ & $d^{-1}$ & 0.1 & 0.03 \\
\hline$R_{\text {denit }}$ & Maximum rate of denitrification at $20^{\circ} \mathrm{C}$ & $d^{-1}$ & 0.3 & 2.0 \\
\hline$K_{\text {denit }}$ & Half-saturation concentration for denitrification & $\frac{m m o l}{m^{3}}$ & 2.0 & 3.0 \\
\hline$F_{\max }^{\text {nit }}$ & Max. sediment flux for nitrate & & -5.0 & -9.55 \\
\hline$K_{\text {sed }}^{\text {nit }}$ & $\begin{array}{l}\text { Half-saturation concentration controlling nitrate } \\
\text { sediment flux }\end{array}$ & $\frac{m m o l}{m^{3}}$ & 100.0 & 173.13 \\
\hline$F_{\max }^{f r p}$ & Max. sediment flux for phosphate & $\frac{m m o l}{m^{2} d^{2}}$ & - & 0.49 \\
\hline
\end{tabular}




$\begin{array}{llll}K_{\text {sed }}^{\text {frp }} & \text { Half-saturation concentration controlling phosphate } \frac{\mathrm{mmol}}{\mathrm{m}^{3}}- & 200.0 \\ & \text { sediment flux } & & \\ \theta_{\text {sed }}^{\text {frp }} & \text { Arrhenius temperature multiplier for phosphate }- & 1.0 & 1.0 \\ & \text { sediment flux }\end{array}$

Table S3 Step-wise model-selection by removing predictors of the multiple linear regression model using seven predictors.

\begin{tabular}{lc}
\multicolumn{1}{c}{ Predictor } & AIC \\
\hline HBR ratio during spring (Spring.HBR) & -61.820 \\
HBR ratio during summer (Summer.HBR) & -60.529 \\
Birgean Work during spring (Spring.Birgean) & -60.189 \\
Gross primary production in the epilimnion (Epi.GPP) & -58.952 \\
Schmidt Stability during summer (Summer.St) & -51.829 \\
Birgean Work during summer (Summer.B) & -50.848 \\
Onset of stratification (Onset.Strat) & -42.900 \\
\hline
\end{tabular}

Table S4 Most parsimonious multiple linear regression model (adjusted $R^{2}=0.84, p<0.001$ ) explaining the summer Anoxic Factor.

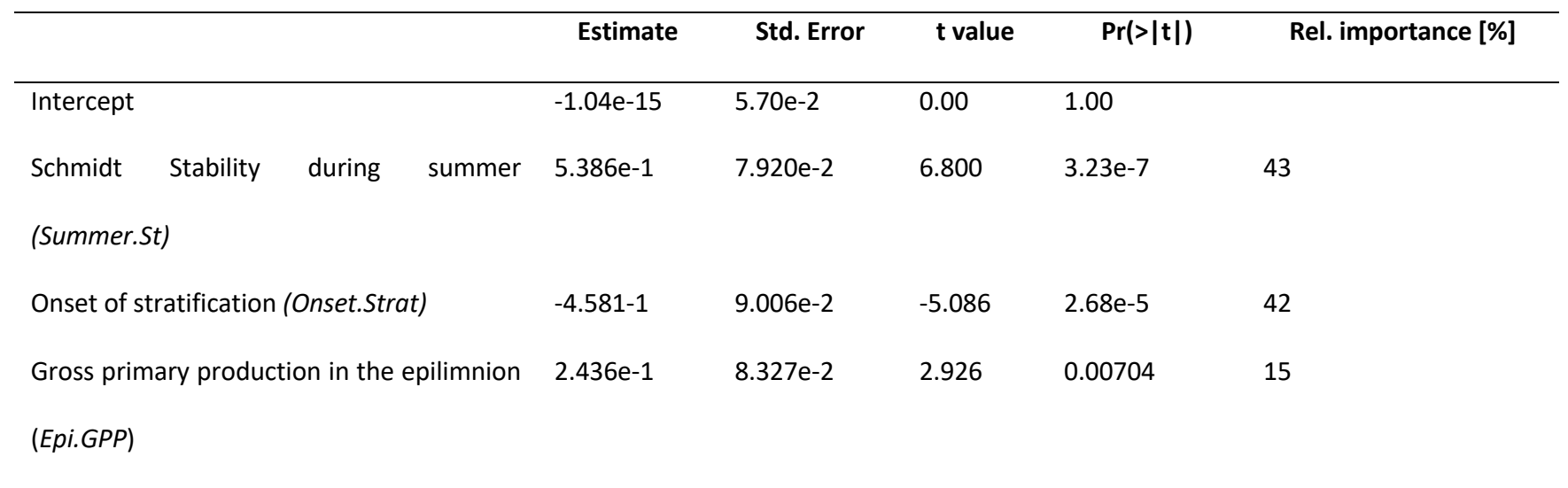

